\title{
Failure mode diagram of rubble pile asteroids: Application to (25143) asteroid Itokawa
}

\author{
Masatoshi Hirabayashi and Daniel J. Scheeres \\ Aerospace Engineering Sciences, 429 UCB, University of Colorado, Boulder, CO 80309-0429 \\ United States \\ email: masatoshi.hirabayashi@colorado.edu
}

\begin{abstract}
Proposing a diagram which shows the variation in asteroidal failure as a function of a spin period, later called the failure mode diagram, this paper considers the failure modes and conditions of asteroid (25143) Itokawa. This diagram is useful to describe when and where failure occurs in an asteroid. Assuming that Itokawa is homogeneous, we use a plastic finite element code to obtain the diagram for this object. The results show that if the bulk cohesive strength is less than $0.1 \mathrm{~Pa}$, Itokawa experiences compressional failure on the neck surface at the current spin period 12.1 hours. At a spin period shorter than 4.5 hours, tension across the neck causes this asteroid to split into two components. It is also found that if the breakup spin period is longer than 5.2 hours, their motion is bounded. This implies that once Itokawa splits, the components may escape from one another.
\end{abstract}

Keywords. Minor planets, asteroids

\section{Introduction}

It has been recognized that the effect of external torques by solar radiation, so-called the YORP effect, plays a crucial role in the rotational evolution of asteroids with less than kilometers in diameter (Rubincam, 2000). Not only has evidence of this effect already been observed in the solar system (Lowry et al., 2007; Taylor et al., 2007; Durech et al., 2008), but it is also predicted that this effect may has strong effects on the formation of the debris disk around a white dwarf (Veras et al., 2014). Recent studies have argued that the YORP effect is sensitive to the obliquity and shape of an asteroid (Vokrouhlicky \& Čapek, 2002; Scheeres, 2007; Statler, 2009) and can possibly change as the body deforms (Cotto-Figueroa et al., 2005). This sensitivity is considered to enrich the orbital evolution of an asteroid (Bottke et al., 2015). Also, because of this effect, some asteroids could reach their spin limits, while others might be rotating at fairly slow spin periods (personal communication with Bill Bottke, 2015).

Observations have also discovered possible candidates of asteroidal activities (failure) by fast rotation. P/2013 R3 has broken into multiple components (Jewitt et al., 2014), 62412 has had dust tails from its nucleus (Sheppard \& Trujillo, 2015), and P/2012 F5 (GIBBS) has ejected dust and fragments (Drahus et al., 2015). These results indicate that the modes of rotational failure may be quite different from one another.

These studies have given rise to the following question: how does the failure mode and conditions change as an irregularly shaped asteroid spins up/down due to the YORP effect? Better understandings of possible deformation/failure at different spin periods help us find clues of it. Analytical and numerical studies have attempted to better understand the fate of spheroidal and elongated asteroids due to fast rotation (Holsapple, 2010; Walsh et al., 2008; Sánchez \& Scheeres, 2012). However, the shape evolution of an 
irregularly shaped asteroid is still poorly understood because, importantly, the YORP effect may cause it to have different failure modes at different spin period.

The purpose of this paper is to propose the failure mode diagram that can describe the variation in the failure modes and conditions of an irregularly shaped asteroid. Providing the minimum cohesive strength that can keep the original shape, later known as the critical cohesive strength, this diagram describes how the original shape fails structurally due to the YORP effect at different spin periods. Here, we focus on the failure modes of asteroid (25143) Itokawa by using the failure mode diagram. The size of Itokawa is $550 \mathrm{~m}$ by $298 \mathrm{~m}$ by $224 \mathrm{~m}$ in diameter, its bulk density is $1.9 \mathrm{~g} \mathrm{~cm}^{-1}$ and the current spin period is $12.1 \mathrm{hr}$ (Fujiwara et al., 2006). In this study, the size and the bulk density are assumed to be constant. Note that Lowery et al. (2014) deduced heterogeneity of the structure from the YORP spin-up. We will consider this exciting problem in the future. To compute plastic deformation of this asteroid we use a plastic finite element model (FEM) first developed by Hirabayashi \& Scheeres (2015).

\section{Modeling}

Hirabayashi \& Scheeres (2015) developed a plastic FEM by extending the technique by Holsapple (2008). The code is implemented by using commercial finite element program ANSYS 15.03. Using a polyhedral shape model of Itokawa (Fujiwara et al., 2006), we create a 10-node tetrahedral FEM mesh, which consists of 48569 nodes and 29633 elements. The rotational and translational motion is canceled out by constraining six node displacements. We improved the original code by Hirabayashi \& Scheeres (2015) to remove the torques and forces caused by misalignment of the center of mass and the moment of inertia axes. This allows the simulations to have better convergence even at more severe conditions.

A material is assumed to be elastic-perfectly plastic and to follow an associated flow rule. The yield condition used is the Drucker-Prager yield criterion, which is described as (Chen \& Han, 1988):

$$
f=\alpha I_{1}+\sqrt{J_{2}}-s \leqslant 0
$$

where $I_{1}$ and $J_{2}$ are the stress invariants, and $\alpha$ and $s$ are functions of a friction angle $\phi$ and cohesive strength $Y$. Here, $\alpha$ and $s$ are defined such that the Drucker-Prager yield surface touches the Mohr-Coulomb yield surface at the compression meridian in the principal stress space. Then, they are given as (Chen \& Han, 1988)

$$
\alpha=\frac{2 \sin \phi}{\sqrt{3}(3-\sin \phi)}, \quad s=\frac{6 Y \cos \phi}{\sqrt{3}(3-\sin \phi)} .
$$

In our code, the total force acting on a tetrahedral element is divided into four parts. Considering all the elements in the body, we compute proper body forces for the nodes at the tips of each element. In this paper, Itokawa is assumed to be affected only by centrifugal and self-gravitational forces. We also consider that the loading ramps up linearly as the simulation goes. This technique was verified by Hirabayashi \& Scheeres (2015).

\section{Failure mode diagram}

To introduce possible failure modes and conditions of an irregularly shaped asteroid effectively we introduce the failure mode diagram. Providing the critical cohesive strength, this diagram describes how an asteroid fails structurally at different spin periods. To 
create this diagram we start from calculating the critical cohesive strength. To find it at a given spin period we initially put relatively high cohesive strength into the plastic FEM code as an input parameter and gradually decrease its value until the simulation reaches computational difficulty because of strong plastic deformation. The solution at the critical cohesive strength is stored to review the detailed deformation modes. We conduct this process at different spin periods.

In addition to the critical cohesive strength at different spin periods, we also calculate the condition of Hill stability to deduce the motion of the split components after an asteroid's breakup. This condition, obtained by considering when the total energy in the system becomes zero, indicates whether or not the orbital motion of the split components is bounded (Scheeres, 2002). Note that the detailed location of the body split can be predicted by looking at propagation of plastic deformation. If the Hill stability condition is slower than the breakup condition, the split components can escape from one another once an asteroid splits.

\section{Results}

We investigate asteroid (25143) Itokawa. The friction angle, Young's modulus and Poisson's ratio are fixed at $35^{\circ}, 1 \times 10^{7} \mathrm{~Pa}$ and 0.25 , respectively (Lambe \& Whitman, 1969). Note that since we do not take into account elastic deformation in the failure model diagram, the value of Young's modulus does not play a role in change of plastic deformation.

We first show the stress distribution at different spin periods in Figure 1. The $x$ axis is taken along the horizontal axis, the $y$ axis aligns downward, and the $z$ axis satisfies the orthogonality with the other axes. The contour plots show the distribution of the normal stress along the $x$ axis on the cross section along the $x$ and $y$ axes. For the contours, negative and positive values describe compression and tension, respectively. Figure 1(a) shows the case of a spin period of 12.1 hours, while Figure 1(b) describes that of 2 hours. At a spin period of 12.1 hours, Itokawa experiences compression everywhere. The middle region has slightly stronger compression than the end regions. On the other hand, at a spin period of 2 hours, the body, especially the neck region, has strong tension.

Plastic regions in Itokawa are described in Figure 2. Figures 2(a) and (b), and (c) and (d) provide the cases of spin periods of 12.1 hours, and 2 hours, respectively. The left and right columns describe the solutions of the surface regions and the cross section, respectively. The contour colors show the stress ratio, a ratio of the current stress to the yield stress. If this ratio has a unity value, the region should experience plastic deformation.

At a spin period of 12.1 hours, the failed region only appears on the surface. The failure mode is strong compression on the surface around the neck. The critical cohesive strength of this spin period is $0.1 \mathrm{~Pa}$. At a spin period of 2 hours, the locations of the failed regions on the surface are almost identical to the 12.1-hour spin period case. However, tensional failure spreads out across the neck region. This implies that the failure mode at this spin period causes this asteroid to stretch along the $x$ axis and to split into the head and the body eventually. The critical cohesive strength at this spin period is $40 \mathrm{~Pa}$.

We second show the failure mode diagram of Itokawa in Figure 3. The critical cohesive strength is given by the solid curve as a function of a spin period. The critical cohesive strength increases exponentially as the spin period becomes shorter. The condition at which tension spreads out across the neck is 4.5 hours and divides the failure modes into two specific modes. At a spin period longer than 4.5 hours, the failure mode is a 


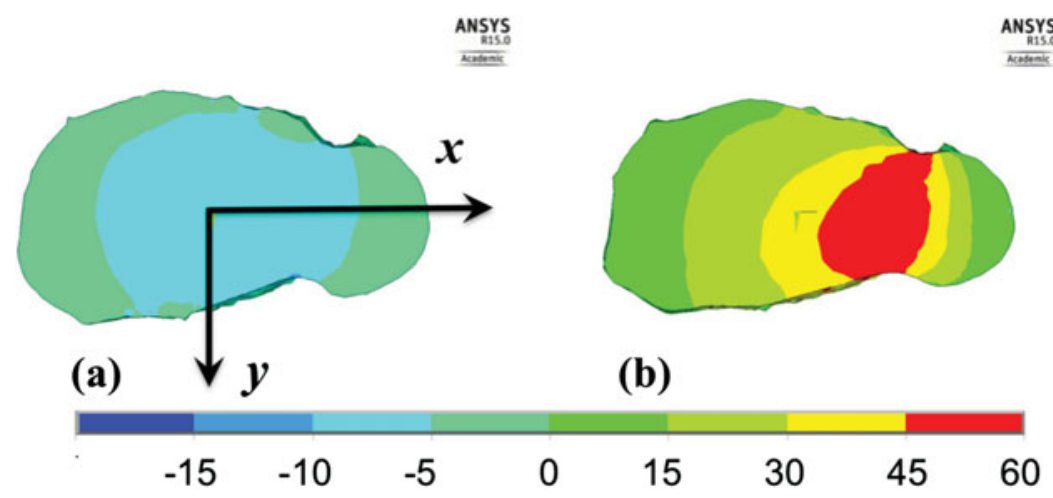

Figure 1. Distribution of the normal stress along the $x$ axis. The numbers in the legend describe pascals. (a) describes the case for a spin period of 12.1 hours, while (b) does that of 2 hours. The color version is available online.

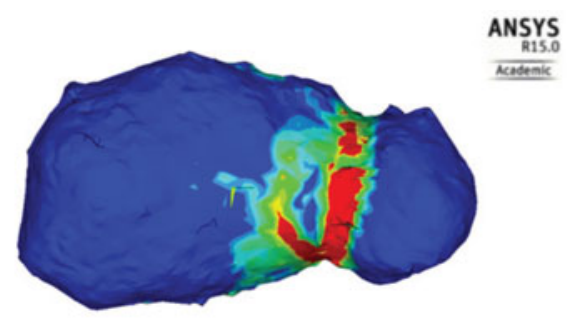

(a)

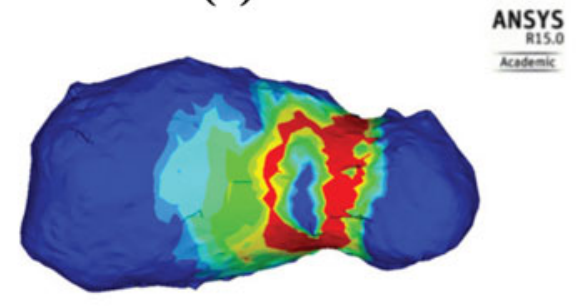

(c)

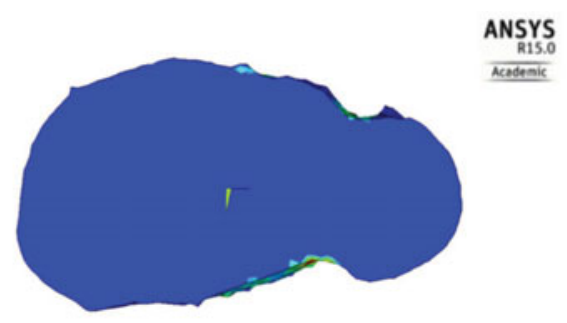

(b)

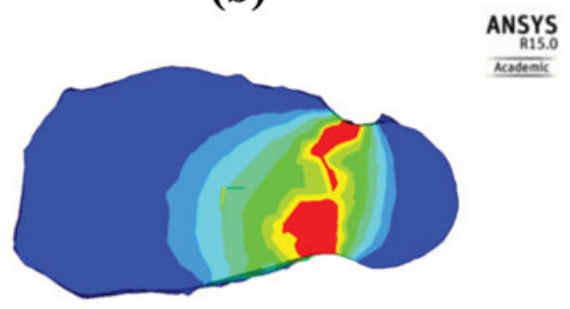

(d)

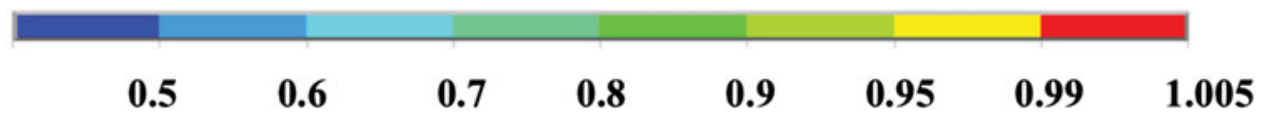

Figure 2. Distribution of the stress ratio in the body of Itokawa. If this ratio is one, the region should fail. (a) and (b) show the case of a spin period of 12.1 hours, while (c) and (d) describe that of 2.0 hours. The left column describes the surface, and the right column gives the cross section. The axes defined here are the same as Figure 1. The color version is available online.

combination of compression and tension of the neck. Because of this, the head and the body do not detach from one another. On the other hand, if Itokawa fails at a spin period shorter than 4.5 hours, it is likely that depending on the bulk cohesive strength, it splits into multiple components. Considering the total energy, we also calculate the Hill stability condition as 5.2 hours. This indicates that once Itokawa breaks into the components, the components may escape from one another. 


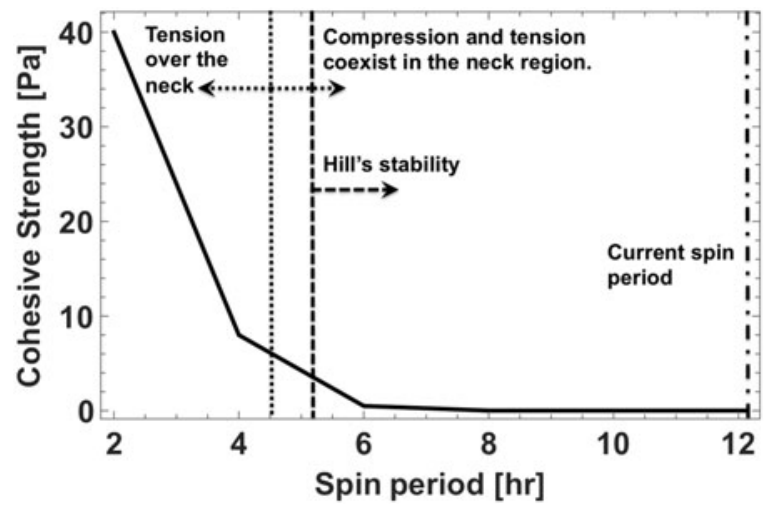

Figure 3. Failure mode diagram of Itokawa. The solid curve shows the critical cohesive strength. The Hill stability condition is 5.2 hours (the dashed line), while the asteroid experiences tension across the neck at a spin period of 4.5 hours (the dotted line).

\section{Discussion and conclusion}

The failure mode diagram can help us better understand failure mechanisms of an irregularly shaped asteroid. By looking at this diagram, we can find when and how an irregularly shaped asteroid fails structurally. In this study, assuming that the structure is homogeneous, we focused on the failure mode diagram of Itokawa. If Itokawa fails at a spin period longer than 4.5 hours, its failure mode is caused by a combination of compression and tension, and does not cause body splitting. However, if Itokawa fails at a spin period shorter than 4.5 hours, it is likely that it fails structurally and splits into the body and the head, which escape from one another. Interestingly, this 4.5-hour condition is much slower than the reported gravitational limit, $\sim 2$ hours (Pravec et al., 2006). This comes from the fact that the neck region is more sensitive to failure than other regions, and thus the shape could easily reshape at slower spin periods. As a result, such rotational evolution may prevent this asteroid from spinning up fast.

It should be noted that although we developed the present model as simple as possible, it is necessary for us to improve two key physical expressions in our code. First, we assumed that $\alpha$ and $s$ are described in Equation (2.2). A choice of $\alpha$ and $s$ may be a crucial point to determine the failure modes of irregularly shaped asteroids. An alternative choice of these parameters is given such that the Drucker-Prager yield surface intersects the Mohr-Coulomb yield surface at the tension meridian (Chen \& Han, 1988). Also, Holsapple (2013) proposed the way which considers a pure shear state superposed on a pressure. Second, although we assumed an associated flow rule, behavior of a geological material would rather follow a non-associated flow rule. However, the key issue of these arguments is that plastic behavior of a geological material in microgravity is unknown. It is necessary for us to narrow down this problem in the future.

\section{Acknowledgements}

The authors wish to thank Bill Bottke and Martin Jutzi for useful discussion about this paper. This work is supported by NASA NNX14AB08G. The authors have permission from ANSYS to use the images generated by ANSYS APDL in this manuscript.

\section{References}

Bottke, W. F., Vokrouhlickỳ D., Walsh K. J., Delbo M., Michel P., Lauretta D. S., Campins H., Connolly, H. C., Scheeres, D. J., \& Chelsey, S. R. 2015, Icarus, 247, 191 
Chen, W. F. \& Han, D. J. 1988, Plasticity for Structural Engineers (Springer-Verlag)

Cotto-Figueroa, D., Statler, T. S., Richardson, D. C., \& Tanga, P. 2005, The Astrophysical Journal, 803, 25

Drahus M., Waniak W., Tendulkar S., Agarwal J., Jewitt D., \& Sheppard S. S. 2015, The Astrophysical Journal Letters, 802, L8

Ďurech, J, Vokrouhlickỳ, D, Kaasalainen, M, Higgins, D, Krugly, Y. N., Gaftonyuk, N. M., Shevchenko, V. G., Chiorny, V. G., Hamanowa, H, Reddy, V, \& Dyvig, R. R. 2008, Astronomy \& Astrophysics, 489, L25

Fujiwara, A, Kawaguchi, J, Yeomans, D.K., Abe, M, Mukai, T, Okada, T, Saito, J, Yano, H, Yoshikawa, M, Scheeres, D. J., Barnouin-Jha, O., Cheng, A. F., Demura, H., Gaskell, R. W., Hirata, N., Ikeda, H., Kominato, T., Miyamoto, H., Nakamura, A. M., Nakamura, R., Sasaki, S., \& Uesugi, K., 2006 Science, 312, 1330

Hirabayashi, M. \& Scheeres, D. J. 2015, The Astrophysical Journal Letters, 798, L8

Holsapple, K. A. 2008, International journal of Non-Linear Mechanics, 43, 733

Holsapple, K. A. 2010, Icarus, 205, 430

Holsapple, K. A. 2013, Planetary and Space Science, 82, 11

Jewitt, D., Agarwal, J., Li, J., Weaver, H., Mutchler, M., \& Larson, S. 2014, The Astrophysical Journal Letters, 784, L8

Lambe, T. W. \& Whitman, R. V. 1969, Soil Mechanis (John Wiley \& Sons)

Lowry, S. C., Fitzsimmons, A., Pravec, P., Vokrouhlickỳ, D., Boehnhardt, H., Taylor, P. A., Margot, J. L., Galád, A., Irwin, M., Irwin, J., \& Kusnirák, P. 2007, Science, 316, 272

Lowry, S. C., Weissman, P. R., Duddy, S. R., Rozitis, B., Fitzsimmons, A., Green, S. F., Hicks, M. D., Snodgrass, C.,Wolters, S. D., Chesley, S. R., Pittichová, J., \& van Oers, P. 2014, $A \mathscr{E A}, 562, \mathrm{~A} 48$

Pravec, P., Harris, A. W., \& Warner, B. D. 2006 Near Earth Objects, our Celestial Neighbors: Opportunity and Risk, Proceedings IAU Symposium, 236, 167

Rubincam D. P., 2000, Icarus, 148, 2

Sánchez, D. P. \& Scheeres, D. J. 2012, Icarus, 218, 876

Scheeres, D. J. 2002, Celestial Mechanics and Dynamical Astronomy, 83, 155

Scheeres, D. J. 2007, Icarus, 188, 430

Sheppard, S. S. \& Trujillo, C. 2015, The Astronomical Journal, 149, 44

Statler T. S. 2009, Icarus, 202, 502

Taylor, P. A., Margot, J.-L., Vokrouhlickỳ, D., Scheeres, D. J., Pravec, P., Lowry, S. C., Fitzsimmons, A., Nolan, M. C., Ostro, S. J., Benner, L. A. M.., Giorgini, J. D., \& Magri, C. 2007, Science, 316, 274

Veras D., Jacobson, S. A. \& Gänsicke, B. T. 2014, Monthly Notices of the Royal Astronomical Society, 445, 2794

Vokrouhlickỳ, D. \& Čapek, D 2002, Icarus, 159, 449

Walsh, K. J., Richardson, D. C., \& Michel, P. 2008, Nature, 454, 188 